\title{
The influence of time step setting on the CFD simulation result of vertical axis tidal
} current turbine

\author{
Dendy Satrio ${ }^{1, *}$, I Ketut Aria Pria Utama ${ }^{2}$, and Mukhtasor ${ }^{2}$ \\ ${ }^{1}$ Ocean Energy Engineering and Management, Institut Teknologi Sepuluh Nopember, \\ 60111 Surabaya, Indonesia \\ *Email: dendy.satrio15@mhs.na.its.ac.id \\ Phone: +62315948757; Fax: +62315932104 \\ ${ }^{2}$ Faculty of Marine Technology, Institut Teknologi Sepuluh Nopember, 60111 \\ Surabaya, Indonesia
}

\begin{abstract}
This paper investigates the influence of time step setting on the CFD simulation result of vertical axis tidal current turbine. Two main features of time step setting have been studied. The first feature concerns with time step size setting that representing the detailed calculation on each degree in one turbine rotation. The second feature deals with number of time step setting that representing how many turbine rotations need for achieving steadiness results. In this study, two-dimensional (2D) analysis of Computational Fluid Dynamics (CFD) simulation using ANSYS-Fluent code with Sliding Mesh technique is used to solve the incompressible Unsteady ReynoldsAveraged Navier-Stokes equations. The finite volume discretization method with second-order scheme and the SIMPLE algorithm are used for all transport equations. Firstly, the numerical model has been validated with available experimental data. Some given scenarios of time step setting for vertical axis tidal turbine have been modeled subsequently. Several increment angle $\left(1^{\circ}, 5^{\circ}, 10^{\circ}\right.$ and $\left.20^{\circ}\right)$ are investigated and chosen the best value of them. Afterwards, the best increment angle combining with several number of rotations (2, 4, 6 and 8 rotations) are investigated. The results show that the more specific of time step size has the better result but it needs more computational times. The recommendation of time step size is $5^{\circ}$ up to $1^{\circ}$. The steadiness calculation result is achieved at six turbine rotations and the rest has no significant effect.
\end{abstract}

Keywords: Vertical axis turbine; time step size; number of time step; sliding mesh; CFD.

\section{INTRODUCTION}

Vertical axis tidal current turbine is one of the most important components in a tidal current energy conversion system [1]. At the first process of energy conversion, the kinetic energy that contained in current movements of sea water is extracted to be mechanical energy by the turbine. This process determines the overall efficiency of the system. Therefore it becomes one of the important challenges in research on a vertical axis tidal current turbine for enhancing the performance [2].

Some studies for enhancing the performance of vertical axis tidal current turbine have been conducted previously by analytical, experimental and numerical methods [39]. Numerical method can be done by simulation using Computational Fluid Dynamics 
software $[5-7,10]$. One of the most familiar software is ANSYS-Fluent code. The code can simulate the turbine operation in rotating or dynamic conditions using Sliding Mesh technique. In the solver setup, there is an adjustable time step setting that can influence the vertical axis tidal current turbine simulation result. Two main features of time step setting are time step size and number of time step. The time step size represents the detailed calculation on each degree of one turbine rotation. The number of time step represents how many turbine rotations need for achieving steadiness result.

Bouzaher et al., 2017 [4] studied and analyzed the influence of flexible blades on the vertical axis tidal turbine performance. There were five airfoil types with different effect of flexibility that studied. The research was conducted by 2D CFD using ANSYS-Fluent 15.0 code. The mesh density was 142,000 cells. The time step size was set to $10^{-4}$ and the steadiness was obtained after three to four of rotor rotations. In this paper, the time step setting was not explained clearly. They said that the time step size was selected after some test of simulations.

Satrio et al., 2017 [5] studied about numerical investigation of contra-rotating vertical axis tidal current turbine performance. The modification of $\mathrm{H}$-Darrieus turbine to be a contra-rotating mode was an effort to improve the turbine performance. They had investigated using 2D CFD with ANSYS-Fluent code. The total mesh was 127,677 cells. The time step size that represented by increment angle was set to $10^{\circ}$ and steadiness was achieved on over three to four rotor rotations. In this paper, how the way to setup the time step setting was not explained. They informed that the time step had been set in such a way.

Torresi et al., 2013 [6] had done numerical investigation of a Darrieus rotor that applicable for low-head hydropower generation. They performed 2D CFD simulation using ANSYS-Fluent code. The mesh density was globally 111,000 cells. The time step size was set to $1^{\circ}$ and each time was performing 75 iterations. The number of rotations was set to 50 of rotor rotations and the result reached periodically after 30 rotations. In this paper, the effect of time step setting on the simulation result was not explained.

Maitre et al., 2013 [7] studied numerical modeling of flow in a Darrieus water turbine. They analyzed the influence on wall grid refinement and compared with experiments. 2D CFD modeling had been done for representing the actual 3D flow in the turbine. The mesh density was set to over eight million cells. They had studied five time steps and they recommended that the increment angle was set to less than $2^{\circ}$ of rotation. The number of rotations was set to 11 rotations and steadiness was obtained after three to four rotor rotations. In this paper, they had been studied some influences of the time step setting on the simulation result but they did not explained how to set the time step in the simulation solver setup. The best time step setting was used in the final simulation setup to gain a good result. Furthermore, the using many mesh density could be effect in the long of computational time. This study did not report how long the computation had been done.

This current study discusses the effect of time step setting on the CFD simulation result of vertical axis tidal current turbine. Detail information about how to set the time step size and the number of time step has been explained at the KEY PARAMETERS section. NUMERICAL SIMULATION section discusses details methodology on 2D CFD simulation. The influence of time step setting on the coefficient of performance error and the computational time have been analyzed and presented in one graphic at RESULTS and DISCUSSION section. The total computational time is dependent on the owned computer capability and the number of mesh. That can effect on the cost of computation. Therefore, it is important to setup the time step size and the number of 
time step in the Fluent solver effectively to obtain good results with acceptable accuracy.

\section{KEY PARAMETERS}

\section{Time Step Setting}

In Sliding Mesh technique, turbine can be simulated in dynamic condition so it can rotate and extract energy from the incoming stream. Illustration in Figure 1 explains that turbine will rotate 360 degrees starting from its first position then rotating and will back on the same position based on the centre axis for achieving one full rotation. The computational calculation will be done on each degree position of the turbine rotation. Number of iteration that has been determined will be computed on each position of rotations until it finish or until it achieve convergence criteria. Time step size represents by increment angle $(\theta)$ for each step rotation. Number of time step represents the total of turbine rotations or how many turbine rotations will be simulated.

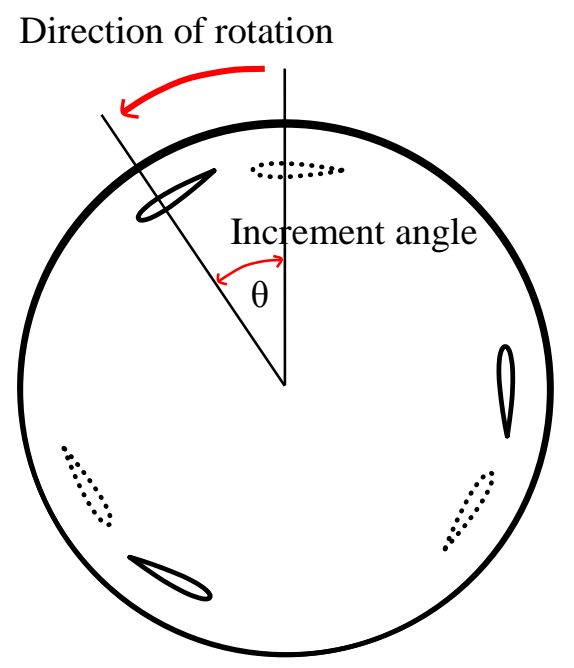

Figure 1. Time step size of each rotation degree.

In the Fluent solver, the time step size (TSS) and the number of time step (NTS) can be set under menu Run Calculation. The equations can be written as follows:

$$
\begin{gathered}
N T S=N \frac{360}{\theta} \\
T S S=\frac{N}{(\omega 0.15915) \times \text { number of time step }}
\end{gathered}
$$

where $N$ is the number of rotations (the value is determined by user), $\theta$ is the increment angle or time step rotation degree (the value is determined by user), $\omega$ is the turbine rotation speed (rad/s), 0.15915 is a constant to convert rad/s unit to rot/s unit.

\section{Performance Parameters}

Some performance parameters are defined to evaluate the effect of time step setting on the simulation results that they can be written as follows:

$$
C_{p}=\lambda C_{m}
$$




$$
\begin{aligned}
\lambda & =\frac{\omega R}{V} \\
C_{p \text { error }} & =\frac{C_{p \text { num }}-C_{p \text { exp }}}{C_{p \exp }} 100 \%
\end{aligned}
$$

where $C p$ is coefficient of performance that a non-dimensional parameter used to evaluate turbine power output, $\mathrm{Cm}$ is coefficient of moment that resulted from simulation output, $\lambda$ is the tip speed ratio that meant ratio between angular speed at blade tip against inlet velocity, $R$ is the turbine radius $(\mathrm{m}), V$ is the inlet velocity $(\mathrm{m} / \mathrm{s})$, $C p$ error is the difference between experimental data ( $C p$ exp) and numerical data $(C p$ num) as indicator to evaluate the effect of different time step setting on the simulation.

\section{NUMERICAL SIMULATIONS}

\section{Computational Domain}

In the current study, the published experimental data of vertical axis turbine by Guang et al., 2013 [9] is used as model geometry reference. As summary in Table 1, main parameters of the turbine geometry are presented. In the CFD simulation, the physical model is changed to be 2D computational model. The 2D turbine model is simplified from the physical model that there are no shaft, arms, and circular plate at end of blades as shown in Figure 2. The computer specifications that used to simulate the 2D CFD analysis are i7-6850 3.6 GHz processor, $64 \mathrm{~GB}$ RAM and 6 GB VGA.

Table 1. Turbine geometry.

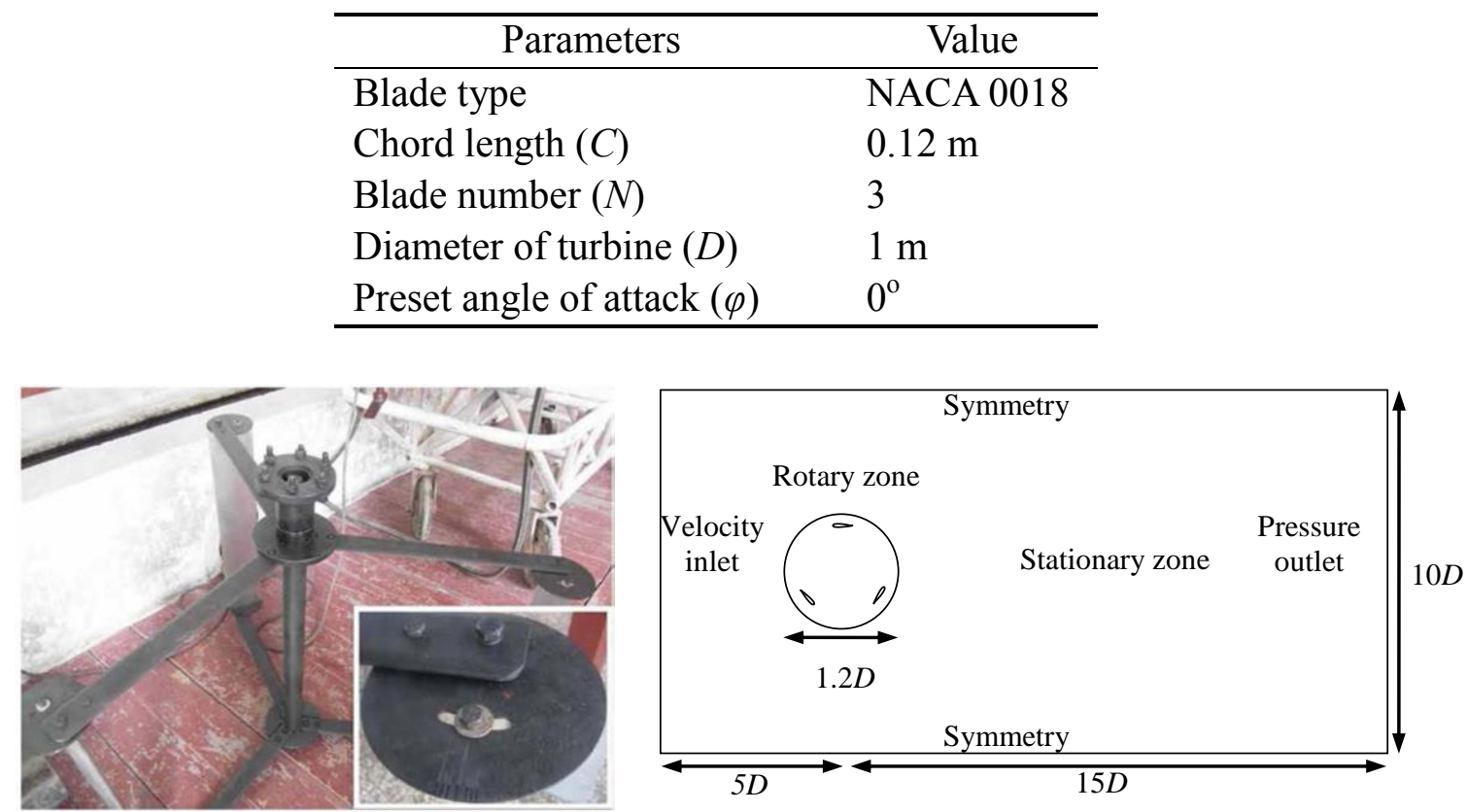

(Model turbine by Guang et al., 2013 [9])

Figure 2. Physical model and 2D turbine model with computational domain.

The computational domain is assigned as $10 D \times 20 D$. This is because for ensuring that blockage effects from wall side is isolated from the turbine and full wake development is achieved far away behind the turbine. In the Sliding Mesh technique, the 
computational domain requires to divide into two zones. They are rotary zone that rotated counter clockwise and stationary zone that fixed in the position. The description of all boundary conditions that used in this simulation are shown in Figure 2. Inlet boundary condition is defined as velocity inlet with a constant value ( $\mathrm{V}=1.5 \mathrm{~m} / \mathrm{s})$. Outlet boundary condition is assigned as pressure outlet with pressure gauge distribution of $0 \mathrm{~Pa}$. Turbulent intensity $5 \%$ and viscosity ratio $10 \%$ as default set up are used in the inlet and outlet boundary conditions. Top and bottom walls are defined as symmetry boundary conditions. The blades specify as wall with no slip conditions and have null velocity relative to the rotary zone.

\section{Mesh Generation}

The computational domain is discretized with hybrid mesh. Triangle meshes are applied in most of the domain but in near the blades wall has refined with quadrilateral mesh to obtain a good result. The counted meshes are 100,015 elements with 68,301 nodes. The mesh density has been chosen due to benchmarking from the prior study by Marsh et al., 2017 [10]. Mesh independence for 2D numerical modeling was achieved starting from 100,000 million elements [10]. As shown in Figure 3, most of the elements (approximately 80\%) is in the region of interface zone and close to the blades. The growth rate has been managed as 1.2 therefore the mesh density changes gradually starting from the rotary domain spread to the stationary domain as depicted in Figure 3. At certain distance, the growth rate will discontinue then the mesh elements have the same size.

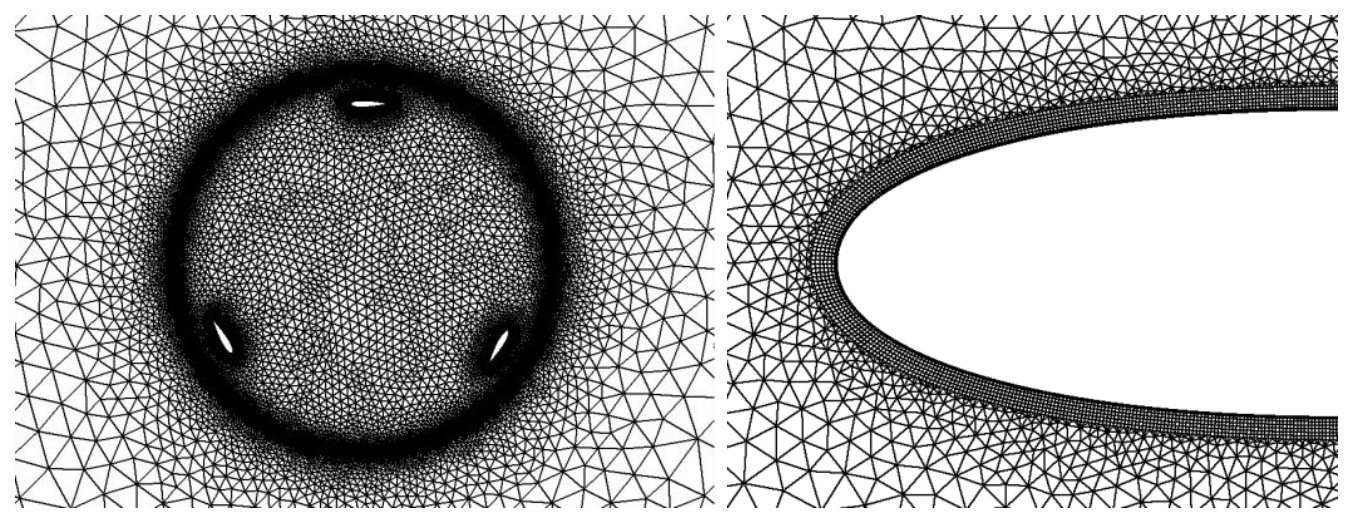

Figure 3. Refinement mesh at interface zone and near the blades wall.

Figure 3 on the right shows the zoom in view of near the blades wall that require to be treated for resolving the boundary layer flows. Inflation layers are applied to control cell height near the blades wall and to set the first height of mesh from the blades surface. In this simulation, Reynolds number are varied from 200,000 up to 500,000. Regarding the Reynolds number value and according to another research, the non-dimensional wall distance $(y+)$ value for the first mesh is 0.99 [10]. After calculated with $y+$ equation, the obtained first mesh height is $1.303110^{-5} \mathrm{~m}$. The second mesh and subsequently will expand until 20 layers with growth rate of 1.2.

\section{Solver Setup}

2D CFD simulation in this study performs by ANSYS-Fluent code. Internal parallel processing with 6 cores is used to solve the simulation. The code is used to solved incompressible Unsteady Reynolds-Averaged Navier-Stokes (U-RANS) equations with 
transient analysis approach. Sliding Mesh technique is used to make the turbine in rotating condition. K- $\omega$ SST two equations of turbulence model are utilized to compute Reynolds stress in the U-RANS equations. K- $\omega$ SST turbulence model is selected because it has good accuracy at the bulk regions and at the boundary layer regions [10]. The SIMPLE algorithm scheme is chosen as the pressure velocity coupling. The finite volume method with least squares cell based is used as spatial discretization gradient. The Reynolds number is approximately 350,000. Therefore this is a turbulent case, all transport equations are suggested to solve using second-order scheme. The turbulent kinetic energy and the specific dissipation rate terms have been discretized by means second order upwind. Second order discretization also used in the momentum and the pressure terms.

The increment angle or time step rotation degree has been determined as $1^{\circ}, 5^{\circ}$, $10^{\circ}$ and $20^{\circ}$. The calculations have been done in 1.6 TSR. Therefore, using Equation 4 can be calculated the turbine rotation is $4.8 \mathrm{rad} / \mathrm{s}$. These scenarios are determined to study the effect of the TSS to the Cp error. Subsequently, the optimal TSS will be chosen and calculated in the different NTS. Number of rotations has been determined as 2, 4, 6 and 8 rotations. As a summary, several scenarios of the time step setting can be seen in Table 2. Number of iterations has been specified as 50. Consequently, the turbine performance at each rotation position will be calculate until 50 iterations or it will be stopped if the iteration process achieved convergence criteria. In this simulation, the convergence criteria are set to $10^{-4}$ for all parameters. The simulation result $(\mathrm{Cm})$ is represented by the average data over the last one rotation. The average $\mathrm{Cm}$ will be used to calculate $C p$ as mentioned at Equation 3.

Table 2. Several scenarios of the time step setting.

(a) Time step size study at 1.6 TSR

\begin{tabular}{rrrrc}
\hline \multicolumn{1}{r}{$\theta$} & $N$ & $\omega$ & \multicolumn{1}{c}{ NTS } & TSS \\
\hline 20 & 4 & 4.8 & 72 & 0.0727 \\
10 & 4 & 4.8 & 144 & 0.0364 \\
5 & 4 & 4.8 & 288 & 0.0182 \\
1 & 4 & 4.8 & 1440 & 0.0036 \\
\hline
\end{tabular}

(b) Number of time step study at 1.6 TSR

\begin{tabular}{rrrrr}
\hline$\theta$ & $N$ & $\omega$ & $N T S$ & $T S S$ \\
\hline 5 & 2 & 4.8 & 144 & 0.0182 \\
5 & 4 & 4.8 & 288 & 0.0182 \\
5 & 6 & 4.8 & 432 & 0.0182 \\
5 & 8 & 4.8 & 576 & 0.0182 \\
\hline
\end{tabular}

\section{RESULTS AND DISCUSSION}

\section{Numerical Validation}

Validation of 2D CFD simulation is carried out against published data by Guang et al., 2013 [9]. The $C p$ curve by red dash line in Figure 4 is obtained from numerical simulation with time step setting of $5^{\circ} \theta$ and $8 N$ or in other words of 576 NTS and 0.0182 TSS. Comparing the numerical result and the experimental data in Figure 4 shows that both of them have similar trend. Even though generally, this numerical model has error in predicted of peak performance and overestimated $C p$ in the all entire $T S R$ domain. This matter is usually happened in 2D CFD modeling [7, 10].

The numerical model proceeds some errors. $C p$ peak value of the numerical model is attained at 2.1 TSR with value of 0.243 whereas $C p$ peak value of the experimental data is attained at 2.269 TSR with value of 0.143 . The $C p$ peak value position is shifted from 2.269 TSR in experimental data to 2.1 TSR in numerical result. In low $T S R$, numerical $C p$ is slightly higher than experimental data. However, numerical 
$C p$ in high TSR is excessively higher than experimental data. The $C p$ peak error value is approximately $70 \%$. Has been realized from the beginning since that this study is using 2D numerical model rather than $3 \mathrm{D}$ model numerical therefore $3 \mathrm{D}$ effect in this simulation cannot be able to capture. The overestimations $C p$ and the errors $C p$ happen because of the neglecting the $3 \mathrm{D}$ effect since in the $2 \mathrm{D}$ numerical model there are no shaft, arms, and circular plate at end of blades. These components can make chaotic flow distribution and produce drag [11]. Furthermore, in a simulation analysis there are several idealizations or assumptions such as neglecting friction and mechanical loss also can cause the overestimation $C p$ [5].

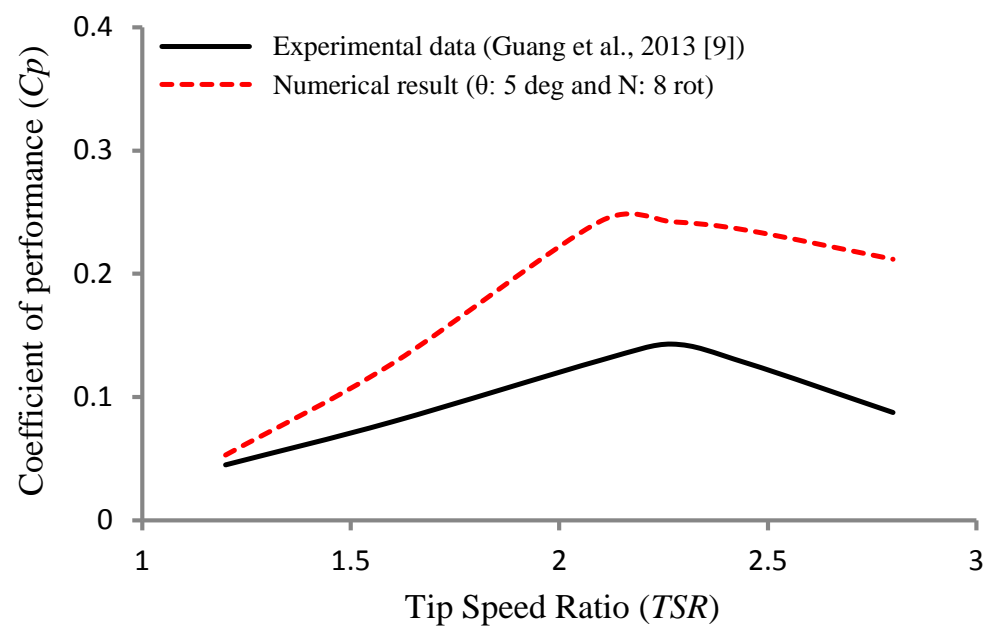

Figure 4. Validation of numerical model with published experimental data.

\section{Time Step Size Study}

The current simulation chooses 1.6 TSR operation condition as a study case then modified in several TSS settings. Several TSS settings can be obtained with defining the increment angles $(\theta)$ are $1^{\circ}, 5^{\circ}, 10^{\circ}$ and $20^{\circ}$ as mentioned in Table 2 . Figure 5 shows the influence of TSS setting toward Cp error that plotted with blue line and computational time that plotted with red dash line.

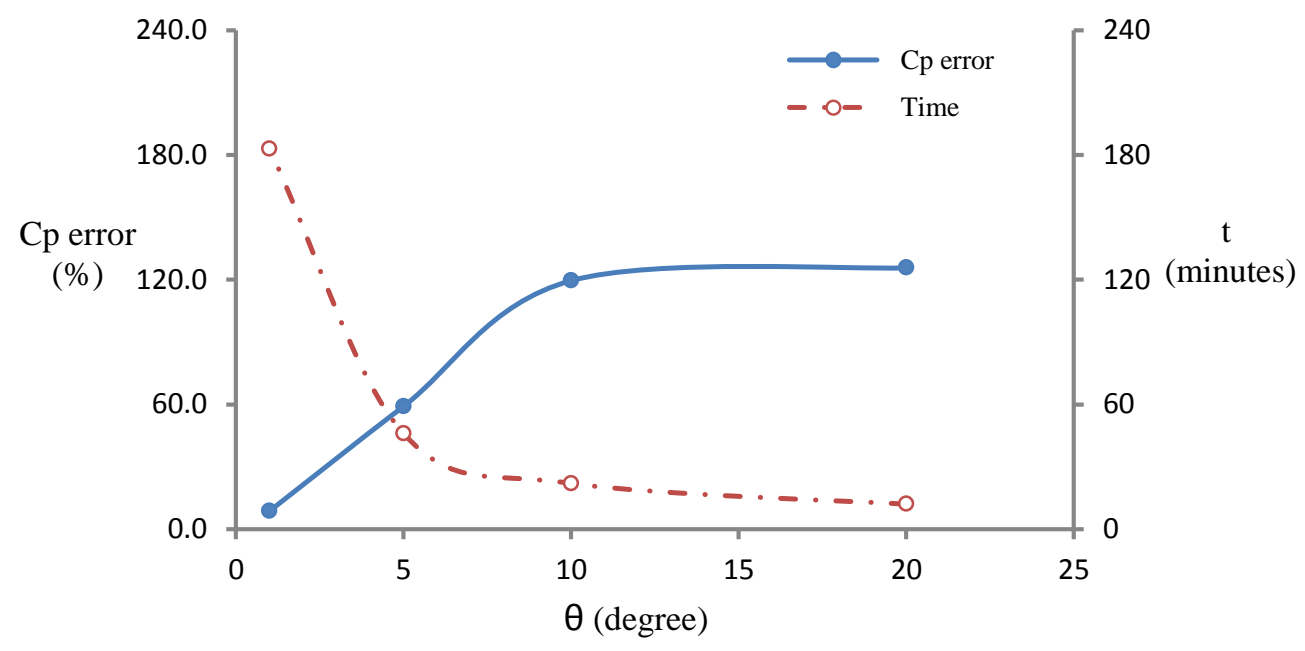

Figure 5. Effect of TSS settings on \% Cp error and computational time at 1.6 TSR. 
Blue line in Figure 5 shows that when the TSS over $10^{\circ}$ resulting $C p$ error until $120 \%$. Therefore TSS over this value is not recommended to apply in the simulation because resulting many errors. It is not recommended even though the computational time consumes just 12 minutes for one operation condition. After that the Cp error will be constant for TSS over $10^{\circ}$. The Cp error will be decrease as decreasing TSS which is below $10^{\circ}$. The best result is achieved at $1^{\circ}$ TSS with $8.7 \%$ Cp error but it needs more computation time up to 183 minutes for one operation condition. $C p$ curves that can be seen in Figure 4 have six points of operation conditions. Consequently, total computational time is approximately 1,098 minutes. This is not efficient because take a long time to solve a problem. In the common sense for CFD researcher that to solve a problem needed many times trials and errors. Therefore, it is recommended to apply time step size around $5^{\circ}$ up to $1^{\circ}$. The $C p$ error can be tolerated and the computational time is not too take a long time. This matter also depends on the computer capability and the total mesh in each calculation case.

\section{Number of Time Step Study}

The increment angle of $5^{\circ}$ is chosen as an optimum value from previous time step size study results. The current simulation is also done in 1.6 TSR operation condition then modified in several NTS settings. Several NTS settings can be gained with defining number of rotations $(N)$ are 2, 4, 6, and 8 rotations. Figure 6 shows the influence of number of rotations setting into $C p$ error and computational time plotted with blue line and red dash line.

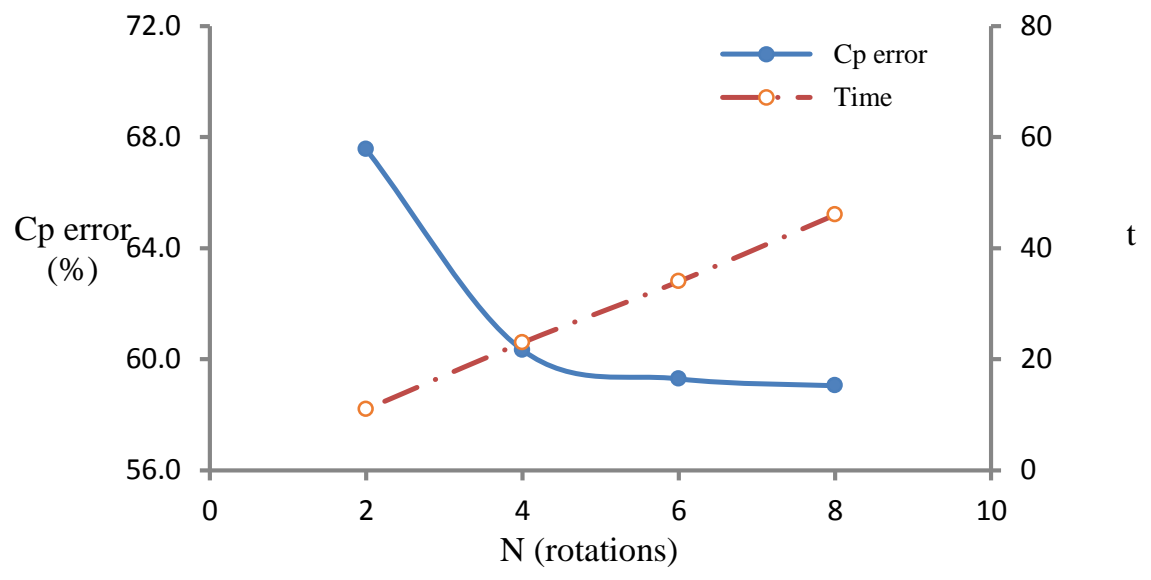

Figure 6. Effect of NTS settings on \% Cp error and computational time at 1.6 TSR.

Cp error curve represented with blue line shows that $N$ below 4 rotations has maximum $C p$ error until $67.6 \%$. Implementation of this setting is not recommended because has many errors. Even though in NTS setting 4 rotations the computation requires only 11 minutes for calculating one operation condition. The $C p$ error will be decrease as increasing NTS over than 6 rotations. The $C p$ error will be relatively constant for NTS around 6 to 8 rotations. NTS settings with $6 N$ and $8 N$ have Cp error of $59.3 \%$ and $59.0 \%$. The Cp error difference is a little but the computational time difference is 12 minutes for calculating one operation condition. In Figure 4 , the $C p$ curve have six operation conditions. Therefore if it multiplied with six operation conditions the total computational time difference can achieve around 72 minutes. 


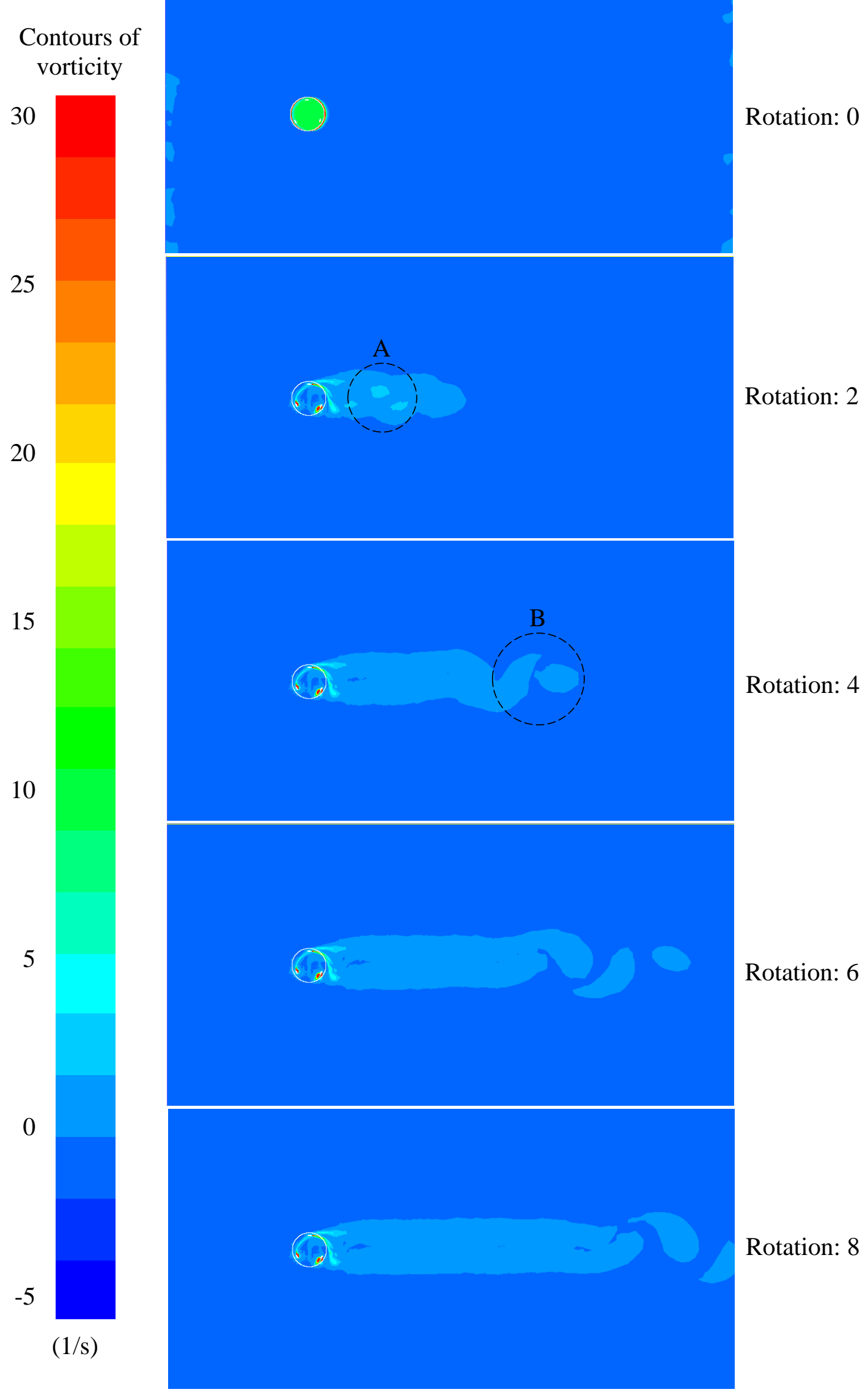

Figure 7. Wake development behind the turbine at 1.6 TSR with $5^{\circ}$ TSS setting. 
Running a simulation process in CFD is not always success. For one case of computational simulation needs a lot of time that gathered from several processes: making geometry model, meshing generation and solver setup. The geometry of numerical model should be drawn like as the physical model and also consider the simulation technique capability. The mesh configuration should be good with enough mesh density for catching the flow field phenomena and fluid interactions between the fluid flow and the blades. In the solver setup itself, there are some configurations included the time step setting that should be proper determined. Therefore, in totally, it requires a lot times to solve one problem.

Furthermore, in turbine dynamic modeling by meant that in rotating conditions, the wake development must achieve full expansion. The turbine performance should be not disturbed by the wake behind the turbine. Figure 7 shows the wake development process at 1.6 TSR with $5^{\circ}$ TSS setting in several NTS settings. At 2 NTS, the wake (Figure 7: A) forms too close behind the turbine. This is explained why 2 NTS has the worst of $C p$ error. In 4 NTS, the full wake development is reached (Figure 7: B). The wake is far away behind the turbine. Therefore, it is recommended to apply NTS starting from 4 or more.

\section{CONCLUSION}

CFD simulations with several time step setting scenarios have been done. The 2D numerical model has been validated with available experimental data. The results show that the recommendation setting of TSS to apply in the simulation is around 5o up to 10 because it has fast computation time and acceptable $\mathrm{Cp}$ error. The full wake development is achieved at fourth turbine rotations. The recommendation setting of NTS is 6 rotations to obtain a simulation result with low Cp error, tolerated computing time consumption and certainty of full wake development.

\section{ACKNOWLEDGEMENTS}

The authors, would like to convey a great appreciation to the Directorate General of Resources for Science, Technology and Higher Education; Ministry of Research, Technology and Higher Education of Republic Indonesia which funding the current project under a scheme called The Education of Master Degree Leading to Doctoral Program for Excellent Graduates (PMDSU) under contract number 135/SP2H/LT/DRPM/IV/2017.

\section{REFERENCES}

[1] Khan MJ, Bhuyan G, Iqbal MT, Quaicoe JE. Hydrokinetic energy conversion systems and assessment of horizontal and vertical axis turbines for river and tidal applications: A technology status review. Applied Energy 2009; 86 1823-1835.

[2] Satrio D, Utama IKAP, Mukhtasor. Vertical Axis Tidal Current Turbine: Advantages and Challenges Review. In: International Conference on Ocean, Mechanical and Aerospace for Scientists and Engineers, Kuala Terengganu, Malaysia, vol.3: pp. 64-71; 2016.

[3] Paraschivoiu I, Trifu O, Saeed F. H-Darrieus Wind Turbine with Blade Pitch Control. International Journal of Rotating Machinery 2009; ID: 505343. 
[4] Bouzaher MT, Guerira B, Hadid M. Performance Analysis of a Vertical Axis Tidal Turbine with Flexible Blades. Journal of Marine Science and Application 2017; 16: 73-80.

[5] Satrio D, Utama IKAP, Mukhtasor. Numerical Investigation of Contra-Rotating Vertical Axis Tidal Current Turbine. Journal of Marine Science and Application 2017.

[6] Torresi M, Fortunato B, Camporeale SM. Numerical investigation of a Darrieus rotor for low-head hydropower generation. In: International Conference on Sustainable Energy Information Technology, Nova Scotiva, Canada, 2013. Procedia Computer Science 19 728-735, 2013.

[7] Maitre T, Amet A, Pellon C. Modeling of the flow in a Darrieus water turbine: Wall grid refinement analysis and comparison with experiments. Renewable Energy 2013, 51 497-512.

[8] Bachant P, Wosnik M. Performance measurements of cylindrical- and sphericalhelical cross-flow marine hydrokinetic turbines, with estimates of exergy efficiency. Renewable Energy 2015, 318-325.

[9] Guang Z, Ran-sheng Y, Yan L, Peng-fei Z. Hydrodynamic performance of a vertical-axis tidal-current turbine with different preset angles of attack. Journal of Hydrodinamics 2013, 25 (2): 280-287.

[10] Marsh P, Ranmuthulaga D, Penesis I, Thomas G. The influence of turbulence model and two- and three-dimensional domain selection on the simulated performance characteristics of vertical axis tidal turbines. Renewable Energy 2017, 105 106-116.

[11] Le TQ, Lee K-S, Park J-S, Ko JH. Flow-driven rotor simulation of vertical axis tidal turbines: A comparison of helical and straight blades. International Journal of Naval Architecture and Ocean Engineering 2014, 6: 257-268. 